\title{
Implications of Feebly Interacting Dark Sector on Neutron Star Properties and Constraints from GW170817
}

\author{
Debashree Sen, ${ }^{1,2 \star}$ Atanu Guha, ${ }^{3} \dagger$ \\ ${ }^{1}$ Department of Physical Sciences \\ Indian Institute of Science Education and Research Berhampur, \\ Transit Campus, Government ITI, Berhampur-760010, Odisha, India \\ ${ }^{2}$ Physics Group, Variable Energy Cyclotron Centre, 1/AF Bidhan Nagar, Kolkata 700064, India \\ ${ }^{3}$ Department of Physical Sciences \\ Indian Institute of Science Education and Research Pune, \\ Dr. Homi Bhabha Road, Ward No. 8, NCL Colony, Pashan, Pune-411008, Maharashtra, India
}

Accepted XXX. Received YYY; in original form ZZZ

\begin{abstract}
We investigate the effect of feeble interaction of dark matter (DM) with hadronic matter on the equation of state (EoS) and structural properties of neutron stars (NSs) in static conditions. For the purpose we adopt the effective chiral model for the hadronic sector and for the first time in the context of possible existence of DM inside NSs, we introduce DM-SM interaction through light new physics mediator. Moreover, the mass of DM fermion, the mediator and the coupling are adopted from the self-interaction constraint from Bullet cluster and from present day relic abundance. Within the considered framework, the work highlights the underlying stiffening of EoS in presence of DM fermion of mass of the order of a few $\mathrm{GeV}$ compared to the no-DM scenario. Consequently, the maximum gravitational mass of NS is obtained consistent with the bounds from the most massive pulsars which were not satisfied with the hadronic matter EoS alone. The estimates of radius and tidal deformability of $1.4 M_{\odot} \mathrm{NS}$ and the tidal deformabilities of the individual components of the binary neutron stars (BNS) associated with GW170817 are all in good agreement with the individual constraints obtained from GW170817 observation of BNS merger.
\end{abstract}

Key words: equation of state; dark matter; neutron star mergers; gravitational waves

\section{INTRODUCTION}

Robust evidences like rotation curves of galaxies, observation of gravitational lensing, $\mathrm{x}$-ray analysis of Bullet cluster affirm the existence of dark matter (DM) in our Universe Bertone et al. (2005); Aghanim et al. (2020). The parameters of the standard model of cosmology are measured using the analysis of the Cosmic Microwave Background (CMB) anisotropy maps. CMB maps are obtained from the Wilkinson Microwave Anisotropy Probe (WMAP) data Ade et al. (2014); Bennett et al. (2013). These measurements and subsequent analysis suggest that the contribution of DM to the total matter content of the Universe to be $\sim 26 \%$, whereas, only $\sim 4 \%$ of the Universe is constituted by the baryonic matter. The understanding of standard cosmology furnishes the present day relic abundances of DM to be $\Omega h^{2} \approx 0.12$ with an uncertainty at the level of $1 \%$ Cannoni (2016). N-body simulations show that the DM profile is Universal i.e., same for all masses Navarro et al. (1996).

Most popular particle candidates for DM are characterized

\footnotetext{
* E-mail: debashree@iiserbpr.ac.in

$\dagger$ E-mail: atanu.guha@students.iiserpune.ac.in
}

by their feeble interaction with the standard model (SM) particles and by their massiveness to generate high gravitational force. The search for such weakly interacting massive particles (WIMPs) are going on over a few decades, specifically following certain avenues. One of them is the direct detection, in which DM particles scatter of nuclei and nuclear/electron recoil energies are measured. Dedicated experiments for direct detection of DM candidates are DAMA/LIBRA Bernabei et al. (2008); Aalseth et al. (2008, 2009), KIMS Lee et al. (2014), CRESST-II Angloher et al. (2016), ZEPLIN Sumner (2005), CRESST-I Bravin et al. (1999), superCDMS Agnese et al. (2018), XENON100 Aprile et al. (2012), XENON1T Aprile et al. (2018), LUX Akerib et al. (2013), PANDAXII Wang et al. (2020), DARKSIDE-50 Agnes et al. (2018), SENSEI Crisler et al. (2018), ADMX Du et al. (2018). Very recently in 2020 the XENON1T observed excess over predicted signal Aprile et al. (2020). The XENON1T collaboration reported an $3.5 \sigma$ excess of events in the electron recoil range of $1 \mathrm{keV}<\mathrm{E}_{\mathrm{R}}<7 \mathrm{keV}$ with 285 events over the backgrounds of $232 \pm 15$ events, with an exposure of 0.65 tonneyears and an unprecedentedly low background Aprile et al. (2020). While the other bins were nearly consistent with the expected background events, the prominent excess events ap- 
peared in the $2-3 \mathrm{keV}$ bins. Another avenue is the indirect detection, where DM particles annihilate via self interaction and SM remnants are looked for. For indirect searches, the leading experiments are FERMI-LAT Atwood et al. (2009), DAMPE Ambrosi et al. (2017), IceCube Aartsen et al. (2014), ATIC Chang et al. (2008), PAMELA Adriani et al. (2009, 2013), AMS-02 Accardo et al. (2014); Aguilar et al. (2014), Voyager1 Boudaud et al. (2017), CALET Adriani et al. (2017, 2018), HAWC Harding \& Dingus (2016), HESS Abdallah et al. (2016), VERITAS Zitzer (2018), MAGIC Elsaesser \& Mannheim (2005), CTA (proposed) Carr et al. (2016). Other avenues include collider searches (where, excess of predicted signals are analyzed to interpret the annihilation of SM particles to produce DM via new physics channel) Aaboud et al. (2019a,b,c,d,e, 2018a,b); Aad et al. (2020a,b,c,d, 2015); Sirunyan et al. (2020a,b, 2019a,b,c,d, 2018a,b); Khachatryan et al. (2016); Alimena et al. (2020); Bhattacherjee et al. (2020); Aaij et al. (2021); Blekman et al. (2020), present day nonbaryonic relic abundances Ade et al. (2014); Bennett et al. (2013) and CMB spectral distortion data by FIRAS and PIXIE Ali-Haïmoud et al. (2015).

Due to having weak interactions with SM particles, WIMPs were in thermal equilibrium with the plasma in early Universe. Assuming a dark matter candidate with mass much less than electroweak scale, one can find that the annihilation of the WIMPs before freeze-out was mediated by weak interactions only and the theoretically predicted relic abundances are in good agreement with the observation of total non-baryonic relic density $(\approx 0.12)$ of the Universe at present day Bauer \& Plehn (2019), which has been obtained from the measurement of CMB anisotropy and the spatial distribution of galaxies Zyla et al. (2020); Tanabashi et al. (2018). In DM phenomenology, this outcome is usually referred to as the WIMP miracle. Any DM model must reproduce this relic density. This requirement sets strong constraints on the model parameters.

In literature there is a wide mass range for DM particle candidates, it is spread from $10^{-22} \mathrm{eV}$ (fuzzy DM)Ni et al. (2019); Bauer et al. (2020); Davoudiasl \& Murphy (2017); Bernal et al. (2018) to $10^{15} \mathrm{GeV}$ Kolb et al. (2007); Kolb \& Long (2017); Alcantara et al. (2019). Pauli exclusion principle forbids the mass of fermionic DM to be less than $25 \mathrm{eV}$ Baltz (2004); Tremaine \& Gunn (1979). However, ideally the candidates of mass below few $\mathrm{keV}$ are too hot for structure formation while for those above $100 \mathrm{TeV}$, the perturbative unitarity violates.

On the other hand, neutron stars (NSs) can be treated as natural laboratories where several branches of physics can be explored. Such branches include the study of matter at extreme conditions of density (5-10 times saturation density) and even beyond the standard practice, be it standard cosmology, gravity or the standard model (SM) of particle physics. The equation of state (EoS) at such high density depends on the composition of NS matter (NSM) which is largely unknown from experimental perspectives. However, few observational and empirical constraints on the structural properties of NS help us to constraint the EoS to certain extent. Massive pulsars like PSR J0348+0432 Antoniadis et al. (2013) and PSR J0740+6620 Cromartie et al. (2019) have put strong upper bounds on the gravitational mass of NSs. With the detection of gravitational wave (GW170817) from binary NS merger (BNSM), stringent constraints on the radius and tidal deformability of a $1.4 M_{\odot}$ NS are also obtained. Moreover, NICER experiment came up with constraints on massradius relationship for PSR J0030+0451 Riley et al. (2019); Miller et al. (2019). Also from the source spectrum analysis of 1E 1207.4-5209 Sanwal et al. (2002) and RX J0720.4-3125 Hambaryan et al. (2017), the maximum bounds on surface redshift are obtained. Any realistic EoS obtained by theoretical modeling of NSM must satisfy the aforesaid constraints on the structural properties of NSs.

NSs are remnants of massive stars that have gone through various stages after the exhaustion of thermo-nuclear fuel. One such prominent stage is the supernova (SN) explosion. Hypothesized DM-SM particles interaction indicates that feasible amount of non-thermal production of DM may take place during the SN explosion. Energy released in a SN explosion is carried away by neutrinos whereas the careful analysis of Kamiokande Hirata et al. (1987) and IMB(IrvineMichigan-Brookhaven) Bionta et al. (1987) data on SN1987A suggests the possible existence of some new physics channel contributing to the SN cooling scenario. But these beyond standard model (BSM) channels are highly constrained Raffelt (1996). Sub-GeV DM particles can contribute to the SN cooling scenario but DM particles of mass more than $100 \mathrm{MeV}$ are phase-space suppressed Kadota \& Silk (2014); Dreiner et al. (2014); Guha et al. (2019, 2017). If we consider $\mathrm{GeV}$ scale DM particles to take part in SN cooling, our current understanding of the cooling mechanism and neutrino signal from SN1987A would be invalidated Janka et al. (2007); Janka (2017). Also the environment could not afford to lose much energy on production of such DM at that stage in order to drive the successful SN explosion. Sub-GeV DM can also get trapped depending on the coupling strength if they do not satisfy the optical depth criteria of free streaming Guha et al. (2019); Shapiro \& Teukolsky (1983). But the amount of such DM trapping can safely be assumed to be small due to much lower production crossection as supported by the direct and indirect detection experiments Essig et al. (2012); Crisler et al. (2018); Agnese et al. (2018); Boudaud et al. (2017); AliHaïmoud et al. (2015). Hence in this work we will consider only thermal DM as a possible constituent for the NSM Panotopoulos \& Lopes (2017); Li et al. (2012b); Bertoni et al. (2013).

NSs can inherit the trapped DM during SN explosion or can accrete Razeira et al. (2011); Perez-Garcia et al. (2010); de Lavallaz \& Fairbairn (2010); Ciarcelluti \& Sandin (2011). They can also create their own DM but the amount is negligible after the Kelvin-Helmholtz cooling phase Bertoni et al. (2013); Nelson et al. (2019). In our present work we considered that the accretion of thermal DM during the NS formation is dominant compared to the other possible sources. Theoretically, the presence of DM in NSs can be explained either by considering the interaction of the DM particles with the baryons (via exchange of Higgs boson) Panotopoulos \& Lopes (2017); Bertoni et al. (2013); Nelson et al. (2019) or without considering any particle interaction between DM and baryonic matter Ellis et al. (2018); Li et al. (2012b). For the former type of treatment, the DM-bayonic matter interaction is implemented in the Lagrangian of the total NS matter including DM and baryonic matter while for the latter type of treatment, the two types of matter interact only gravitationally and the two-fluid method is often successfully adopted Tolos \& Schaffner-Bielich (2015); Deliyergiyev et al. (2019); 
Rezaei (2017); Mukhopadhyay et al. (2017). It is seen that $\mathrm{MeV}-\mathrm{GeV}$ scale DM particles may be capable of playing a key role in BNSM. The dimensionless tidal deformability $(\Lambda)$ is enhanced significantly by the presence of trace amount of DM interacting with baryonic matter inside NSs Bertoni et al. (2013); Nelson et al. (2019). However, in the case where DM does not interact with hadronic matter, the tidal deformability is seen to decrease considerably Ellis et al. (2018) along with mass and radius Li et al. (2012b). In the present work, we consider the former type of treatment where fermionic DM particles $\chi$ interact feebly with baryonic matter $\psi$ via exchange of a light and scalar new physics mediator $\phi$ that mixes with SM Higgs boson. The main aim of the present work is to study the effects of interaction of DM and baryonic matter on NS properties of DM admixed NSs.

In popular models in literature, where DM-baryonic matter interaction is taken into account, DM directly interacts with nucleons via the exchange of SM Higgs Boson, irrespective of its spin Andreas et al. (2008). Fundamentally there is no theoretical justification for that and in principle DM-Higgs coupling can be zero Panotopoulos \& Lopes (2017). In the following discussion ahead, we considered a singlet scalar mediator belonging to new physics, which mixes with SM-Higgs boson and hence be able to interact to the nucleons Krnjaic (2016). Presence of light GeV-scale DM without interactions with the nucleons can actually soften the EoS drastically $\mathrm{Li}$ et al. (2012b), whereas by introducing the interaction term we obtained stiffer EoS (Fig. 2). For the pure hadronic matter sector, we have adopted the effective chiral model Sahu \& Ohnishi (2000); Jha \& Mishra (2008); Sen \& Jha (2019); Sen (2021) for $\beta$ equilibrated NSM. However, for a two-fluid treatment, where interaction between DM and HM is not considered via particle interaction, further realistic EoS for the hadronic matter may also be adopted. For example, EoS obtained by interpolating high and low density domains and constrained by the inputs from low-energy nuclear physics, high-density limit from perturbative QCD, and observational data of pulsars Kurkela et al. (2014), is successfully adopted to describe NS configurations in the presence of DM with the help of two-fluid approach Tolos \& Schaffner-Bielich (2015); Deliyergiyev et al. (2019).

Our discussion is organized as follows. In Sec. 2 we present the basic details of the model in presence of DM and the parameter sets we considered. Numerical results and subsequent discussions can be found in Sec. 3. Finally we conclude in Sec. 4.

\section{FORMALISM}

\subsection{Model including Higgs portal dark sector}

We consider the effective chiral model Sahu \& Ohnishi (2000); Jha \& Mishra (2008); Sen (2021) for the hadronic matter sector. The model is based on chiral symmetry and the interaction of the nucleons $\psi$ with the scalar $\sigma$, vector $\omega$, isovector $\rho$ and the pseudoscalar $\pi$ mesons as mediators. In addition to the Lagrangian density for the effective chiral model given in Sahu \& Ohnishi (2000); Jha \& Mishra (2008); Sen (2021), we propose to include the following renormalizable operator to incorporate the new physics (feebly interacting dark sector)

$$
\mathcal{L}_{\text {new }}=\partial_{\mu} \Phi \partial^{\mu} \Phi-\mu_{\Phi}^{2} \Phi^{\dagger} \Phi-\lambda_{\Phi}\left(\Phi^{\dagger} \Phi\right)^{2}-\lambda_{m i x} \Phi^{\dagger} \Phi H^{\dagger} H
$$

where, $\Phi$ is the singlet scalar mediator of mass $m_{\phi}$. For this theory we can diagonalize the scalar mass terms (Eq.(1)) after electroweak symmetry breaking. The resulting mass eigenstates are identified as follows: $\phi$ be the DM-SM mediator and $h$ the Higgs boson Krnjaic (2016). Invoking this, total Lagrangian density becomes

$$
\mathcal{L}=\mathcal{L}_{\text {hadron }}+\mathcal{L}_{d m}
$$

where,

$$
\mathcal{L}_{d m}=\frac{1}{2} \partial_{\mu} \phi \partial^{\mu} \phi-\frac{1}{2} m_{\phi}^{2} \phi^{2}-\frac{1}{4} \lambda_{\phi} \phi^{4}+\bar{\chi}\left[i \gamma_{\mu} \partial^{\mu}-m_{\chi}\right] \chi-y_{\phi} \phi \bar{\chi} \chi
$$

where, $\chi$ is the DM fermion of mass $m_{\chi}$ and it couples to the scalar mediator $\phi$ via Yukawa type coupling with coupling strength $y_{\phi}$. Also the new mediator acquires a coupling to SM fermions as it mixes with the Higgs boson. Expanding in mass basis, we obtain the mediator-SM interaction

$$
\mathcal{L}_{\phi, S M}=\sum_{f} g_{f} \bar{f} f \phi
$$

where, $f$ be the SM fermions of mass $m_{f}$ and the corresponding coupling

$$
g_{f}=\frac{m_{f}}{v} \sin \theta
$$

$\theta$ be the mixing parameter and $v=246 \mathrm{GeV}$ be the vacuum expectation value of the Higgs boson. From the coupling of the $\phi$ to the quarks we can estimate the effective coupling of the $\phi$ to the nucleons, let us call it $g_{\phi N N}$ (details given in the appendix).

Now taking the contribution of the SM and BSM particles, the total Lagrangian density becomes

$$
\begin{aligned}
\mathcal{L} & =\bar{\psi}\left[\left(i \gamma_{\mu} \partial^{\mu}-g_{\omega} \gamma_{\mu} \omega^{\mu}-\frac{1}{2} g_{\rho} \overrightarrow{\rho_{\mu}} \cdot \vec{\tau} \gamma^{\mu}\right)-g_{\sigma}\left(\sigma+i \gamma_{5} \vec{\tau} \cdot \vec{\pi}\right)-g_{\phi N N} \phi\right] \psi \\
& +\frac{1}{2}\left(\partial_{\mu} \vec{\pi} \partial^{\mu} \vec{\pi}+\partial_{\mu} \sigma \partial^{\mu} \sigma\right)-\frac{\lambda}{4}\left(x^{2}-x_{0}^{2}\right)^{2}-\frac{\lambda B}{6}\left(x^{2}-x_{0}^{2}\right)^{3}-\frac{\lambda C}{8}\left(x^{2}-x_{0}^{2}\right)^{4}-\frac{1}{4} F_{\mu \nu} F^{\mu v}+\frac{1}{2} g_{\omega}^{2} x^{2} \omega_{\mu} \omega^{\mu}-\frac{1}{4} \vec{R}_{\mu \nu} \cdot \vec{R}^{\mu \nu}+\frac{1}{2} m_{\rho}^{2} \vec{\rho}_{\mu} \cdot \vec{\rho}^{\mu} \\
& +\frac{1}{2} \partial_{\mu} \phi \partial^{\mu} \phi-\frac{1}{2} m_{\phi}^{2} \phi^{2}-\frac{1}{4} \lambda_{\phi} \phi^{4}+\bar{\chi}\left[i \gamma_{\mu} \partial^{\mu}-\left(m_{\chi}+y_{\phi} \phi\right)\right] \chi
\end{aligned}
$$

We have the following relations from chiral symmetry 


$$
\begin{aligned}
m & =g_{\sigma} x_{0}+g_{\phi N N} \phi_{0} ; \quad m_{\sigma}=\sqrt{2 \lambda} x_{0} ; \quad m_{\omega}=g_{\omega} x_{0} \\
\langle\sigma\rangle & =x_{0} ; \quad C_{\omega}=\frac{g_{\omega}^{2}}{m_{\omega}^{2}} ; \quad C_{\sigma}=\frac{g_{\sigma}^{2}}{m_{\sigma}^{2}}
\end{aligned}
$$

$B$ and $C$ are coefficients of the higher order scalar field terms. Like Panotopoulos \& Lopes (2017) we have taken attractive potential for the new physics scalar mediator consistent with Hambye \& Vanderheyden (2020); Barbieri \& Curci (1989). Note that in the total Lagrangian, the last term $\bar{\psi}\left(g_{\phi N N} \phi\right) \psi$ of the first line of equation 6 indicates the interaction between the scalar new physics mediator $\phi$ from dark sector and the nucleons $\psi$. The strength of the interaction is determined by the coupling constant $g_{\phi N N}$ which is evaluated in the appendix.

The effective masses of nucleons $\left(m^{\star}\right)$ and dark matter $\left(m_{\chi}^{\star}\right)$ are obtained as

$$
\begin{aligned}
& m^{\star}=g_{\sigma} \sigma+g_{\phi N N} \phi \\
& m_{\chi}^{\star}=m_{\chi}+y_{\phi} \phi
\end{aligned}
$$

The equation of motion for the different mesons, nucleons and the DM can be obtained by applying the mean field treatment. The scalar equation of motion is given in terms of $Y=m^{\star} / m$ as

$$
\left(1-Y^{2}\right)-\frac{B}{C_{\omega}}\left(1-Y^{2}\right)^{2}+\frac{C}{C_{\omega}^{2}}\left(1-Y^{2}\right)^{3}-\frac{2 C_{\sigma} \rho_{s}}{Y\left(m-g_{\phi N N} \phi_{0}\right)}+\frac{2 C_{\sigma} C_{\omega}}{Y^{4}\left(m-g_{\phi N N} \phi_{0}\right)^{2}}=0
$$

while the vector $\omega$ and isovector $\rho$ meson field equations remain unchanged and same as obtained without including DM Sahu \& Ohnishi (2000); Jha \& Mishra (2008) viz.

$$
\omega_{0}=\frac{\rho}{g_{\omega} x^{2}}
$$

and

$$
\rho_{03}=\sum_{N} \frac{g_{\rho}}{m_{\rho}^{2}} I_{3_{N}} \rho_{N}
$$

Here ' 3 ' denotes for the third component in isospin $I$ of the individual nucleons $N$.

The scalar density is given as

$$
\rho_{S}=\langle\bar{\psi} \psi\rangle=\frac{\gamma}{2 \pi^{2}} \int_{0}^{k_{F}} d k k^{2} \frac{m^{\star}}{\sqrt{k^{2}+m^{\star}}}
$$

while the baryon density as

$$
\rho=\left\langle\psi^{\dagger} \psi\right\rangle=\frac{\gamma}{2 \pi^{2}} \int_{0}^{k_{F}} d k k^{2}
$$

Here the total baryon density is the sum of individual nucleon densities i.e., $\rho=\rho_{n}+\rho_{p}$.

Since in the mean field treatment we have $\langle\pi\rangle=0$ with $m_{\pi}=0$, the explicit contribution of the pions is not taken into account.

The equation of motion of the DM-SM mediator field is given as

$$
\phi_{0}=\frac{m_{\chi}^{\star}-m_{\chi}}{y_{\phi}}
$$

\subsection{Equation of State}

The EoS viz. the energy density $\varepsilon$ and pressure $P$ is calculated by calculating the energy-momentum tensor involving the Lagrangian 6 . The total energy density and total pressure is computed as

$$
\begin{gathered}
\varepsilon=\frac{\left(m-g_{\phi N N} \phi_{0}\right)^{2}\left(1-Y^{2}\right)^{2}}{8 C_{\sigma}}-\frac{\left(m-g_{\phi N N} \phi_{0}\right)^{2} B\left(1-Y^{2}\right)^{3}}{12 C_{\sigma} C_{\omega}}+\frac{\left(m-g_{\phi N N} \phi_{0}\right)^{2} C\left(1-Y^{2}\right)^{4}}{16 C_{\sigma} C_{\omega}^{2}}+\frac{C_{\omega} \rho^{2}}{2 Y^{2}} \\
+\frac{1}{2} m_{\rho}^{2} \rho_{03}^{2}+\frac{\gamma}{2 \pi^{2}} \int_{0}^{k_{F}} \sqrt{k^{2}+m^{\star 2}} k^{2} d k+\frac{\gamma}{2 \pi^{2}} \sum_{\lambda^{\prime}=e, \mu} \int_{0}^{k_{\lambda^{\prime}}} \sqrt{k^{2}+m_{\lambda}^{2}} k^{2} d k+\frac{1}{2} m_{\phi}^{2} \phi_{0}^{2}+\frac{1}{4} \lambda_{\phi} \phi_{0}^{4}+\frac{\gamma_{\chi}}{2 \pi^{2}} \int_{0}^{k_{F}^{\chi}} \sqrt{k_{\chi}^{2}+m^{\star 2}} k_{\chi}^{2} d k_{\chi} \\
P=-\frac{\left(m-g_{\phi N N} \phi_{0}\right)^{2}\left(1-Y^{2}\right)^{2}}{8 C_{\sigma}}+\frac{\left(m-g_{\phi N N} \phi_{0}\right)^{2} B\left(1-Y^{2}\right)^{3}}{12 C_{\sigma} C_{\omega}}-\frac{\left(m-g_{\phi N N} \phi_{0}\right)^{2} C\left(1-Y^{2}\right)^{4}}{16 C_{\sigma} C_{\omega}^{2}}+\frac{C_{\omega} \rho^{2}}{2 Y^{2}} \\
\quad+\frac{1}{2} m_{\rho}^{2} \rho_{03}^{2}+\frac{\gamma}{6 \pi^{2}} \int_{0}^{k_{F}} \frac{k^{4} d k}{\sqrt{k^{2}+m^{\star 2}}}+\frac{\gamma}{6 \pi^{2}} \sum_{\lambda^{\prime}=e, \mu} \int_{0}^{k_{\lambda^{\prime}}} \frac{k^{4} d k}{\sqrt{k^{2}+m_{\lambda^{\prime}}^{2}}}-\frac{1}{2} m_{\phi}^{2} \phi_{0}^{2}-\frac{1}{4} \lambda_{\phi} \phi_{0}^{4}+\frac{\gamma_{\chi}}{6 \pi^{2}} \int_{0}^{k_{F}^{\chi}} \frac{k_{\chi}^{4} d k_{\chi}}{\sqrt{k_{\chi}^{2}+m^{\star 2}}}
\end{gathered}
$$




\subsection{Model parameter set for hadronic matter}

There are five parameters of the hadronic model given as $C_{i}=$ $g_{i}^{2} / m_{i}^{2}$ where $i=\sigma, \omega, \rho$ and $B$ and $C$. They are determined by reproducing the saturated nuclear matter (SNM) properties. The hadronic model parameter set, chosen for the present work, is adopted from Jha \& Mishra (2008) and is given in table 1 along with the SNM properties yielded by the set.

The values of SNM properties like symmetry energy coefficient $(J=32 \mathrm{MeV})$, saturation density $\left(\rho_{0}=0.153 \mathrm{fm}^{-3}\right)$, binding energy per particle $(B / A=-16.3 \mathrm{MeV})$ and the nuclear incompressibility $(K=231 \mathrm{MeV})$, yielded by the model parameter are well consistent with findings of Stone \& Reinhard (2007); Dutra et al. (2014); Khan \& Margueron (2012, 2013); Garg \& Colò (2018). The value slope parameter $\left(L_{0}=\right.$ $87 \mathrm{MeV}$ ) is also within the range of $L_{0}$ prescribed by Dutra et al. (2014) and comparable with the results of Fattoyev et al. (2018); Zhu et al. (2018) based on the co-relations between the symmetry energy and tidal deformability and radius of a $1.4 M_{\odot} \mathrm{NS}$.

However, as seen from Jha \& Mishra (2008); Jha et al. (2006, 2008) that the EoS yielded by this parameter set, though is in accordance with the heavy-ion collision data Danielewicz et al. (2002), passes through the soft band of heavy-ion collision data for PNM Jha \& Mishra (2008). As discussed in Sahu \& Ohnishi (2000); Jha \& Mishra (2008); Sen \& Jha (2019), this is because of the high value of nucleon effective mass $\left(m^{*}=0.87 \mathrm{~m}\right)$ yielded by the present model compared to other RMF models Horowitz \& Serot (1981); Dutra et al. (2014) and the dominance of vector repulsive force at high densities. It can be seen from table 1 of Jha \& Mishra (2008) that higher ratio of $C_{\sigma} / C_{\omega}$ and more negative values of $B$ give higher effective mass and lower nuclear incompressibility and consequently softer EoS Jha \& Mishra (2008) yielding low mass NS configurations that do not satisfy the maximum mass constraint of NSs Cromartie et al. (2019).

Next we present the parameter sets chosen for the dark matter sector in section 2.4 .

\subsection{Parameter set for dark sector}

Inside the $\mathrm{NS}$ we are not considering non-thermal production of DM fermions $(\chi, \bar{\chi})$. To set some benchmark points, we consider the self interaction constraints coming from bullet cluster to determine the corresponding value of the mass $\left(m_{\phi}\right)$ of the light mediator $\phi$ Tulin et al. (2013); Tulin \& Yu (2018); Hambye \& Vanderheyden (2020). To determine the value of the coupling $y_{\phi}$ we took the help of the present day thermal relic abundances of DM Belanger et al. (2014); Gondolo \& Gelmini (1991); Guha et al. (2019). So in case of non-thermal production this is not stringent.

For DM fermions of mass $m_{\chi}$, self-scattered through a light scalar mediator of mass $m_{\phi}$, the constraint from bullet cluster structure formation is $\sigma_{T} / m_{\chi} \leqslant 1.25 \mathrm{~cm}^{2} / \mathrm{gm}$ Randall et al. (2008); Robertson et al. (2017); $\sigma_{T}$ be the selfscattering transfer cross-section. In figure 1 we show the region $\sigma_{T} / m_{\chi}=(0.1-10) \mathrm{cm}^{2} / \mathrm{gm}$, which is the typical value for known galaxies and clusters Randall et al. (2008); Bradac et al. (2006); Dawson et al. (2012); Dave et al. (2001); Vogelsberger et al. (2012); Kahlhoefer et al. (2015). The coupling $y_{\phi}$ has been fixed from observed relic abundance Belanger et al. (2014). We take $\lambda_{\phi}=0$ in the present work.

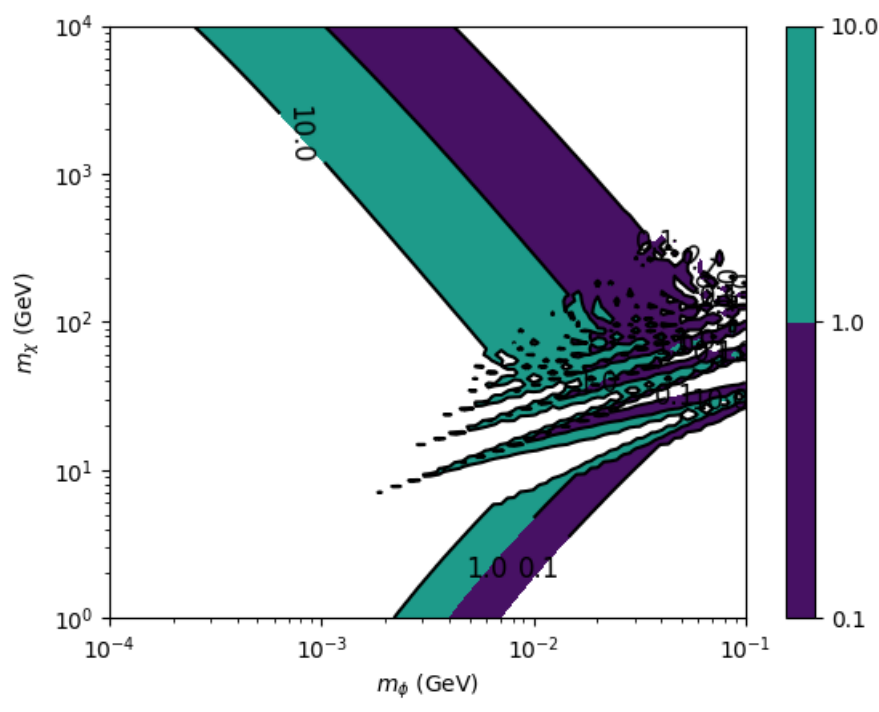

Figure 1. Combination of $m_{\chi}$ and $m_{\phi}$ satisfying the self-interaction constraint from bullet cluster Randall et al. (2008); Tulin et al. (2013). The color coding denotes the values of DM self-interaction $\sigma_{T} / m_{\chi}=(0.1-1) \mathrm{cm}^{2} / \mathrm{gm}$ (violet) and $(1-10) \mathrm{cm}^{2} / \mathrm{gm}$ (green).

For the analysis we have assumed a constant density of fermionic DM throughout the NS and beyond. In our analysis, the value of the DM density is 1000 times smaller than the average neutron number density. DM mass fraction inside the NS is $\sim \mathcal{O}(0.01) \mathrm{Li}$ et al. (2012a); Panotopoulos \& Lopes (2017). Using the SNM number density, the DM number density becomes roughly $\rho_{\chi}=10^{-3} \times \rho_{0} \sim 0.15 \times 10^{-3} \mathrm{fm}^{-3}$. From that we estimated a constant Fermi momentum of dark matter fermions to be $k_{F}^{\chi}=0.033 \mathrm{GeV}$. For $10 \rho_{0}, k_{F}^{\chi}$ is around $0.06 \mathrm{GeV}$. So we varied $k_{F}^{\chi}$ from $0.01 \mathrm{GeV}$ to $0.06 \mathrm{GeV}$.

Considering the aforesaid parameter sets for both hadronic matter and DM, we proceed to compute the EoS and structural properties of NS in presence of DM in the next section. The structural properties of NS are obtained by solving the Tolman-Oppenheimer-Volkoff (TOV) equations Tolman (1939); Oppenheimer \& Volkoff (1939) numerically with the obtained EoS. The dimensionless tidal deformability $(\Lambda)$ can be obtained in terms of the mass, radius and the tidal love number $\left(k_{2}\right)$ following Hinderer (2008); Hinderer et al. (2010); Alvarez-Castillo et al. (2019). The dimensionless tidal deformability of the individual components of the BNS associated with merger corresponding to GW170817 can also be calculated following Raithel et al. (2018); Alvarez-Castillo et al. (2019).

\section{RESULTS AND DISCUSSIONS}

\subsection{Dark matter admixed neutron star for different values of $m_{\chi}$}

Firstly, we compute the DM admixed EoS for different values of $m_{\chi}$ for $k_{F}^{\chi}=0.06 \mathrm{GeV}$. The result is depicted in figure 2 .

For better comparison, we have also shown the $\operatorname{EoS}$ for $\beta$ equilibrated NSM without DM as 'H'. It is seen that the EoS stiffens considerably when the presence of DM is considered compared to the pure hadronic EoS. With the decrease in DM mass, further feeble stiffening is noticed. Contrary to the 
Table 1. Parameter set for the hadronic model (adopted from Jha \& Mishra (2008)) along the saturation properties.

\begin{tabular}{cccccccccccc}
\hline \hline $\begin{array}{c}C_{\sigma} \\
\left(\mathrm{fm}^{2}\right)\end{array}$ & $\begin{array}{c}C_{\omega} \\
\left(\mathrm{fm}^{2}\right)\end{array}$ & $\begin{array}{c}C_{\rho} \\
\left(\mathrm{fm}^{2}\right)\end{array}$ & $\begin{array}{c}B / m^{2} \\
\left(\mathrm{fm}^{2}\right)\end{array}$ & $\begin{array}{c}C / m^{4} \\
\left(\mathrm{fm}^{4}\right)\end{array}$ & $Y$ & $\begin{array}{c}m_{\sigma} \\
(\mathrm{MeV})\end{array}$ & $\begin{array}{c}f_{\pi} \\
(\mathrm{MeV})\end{array}$ & $\begin{array}{c}K \\
(\mathrm{MeV})\end{array}$ & $\begin{array}{c}B / A \\
(\mathrm{MeV})\end{array}$ & $\begin{array}{c}J\left(L_{0}\right) \\
(\mathrm{MeV})\end{array}$ & $\begin{array}{c}\rho_{0} \\
\left(f m^{-3}\right)\end{array}$ \\
\hline \hline 7.325 & 1.642 & 5.324 & -6.586 & 0.571 & 0.87 & 444.614 & 153.984 & 231 & -16.3 & $32(88)$ & 0.153 \\
\hline \hline
\end{tabular}

Table 2. Chosen values of self interacting DM $m_{\chi}$ and corresponding values of $m_{\phi}$ from the constraints obtained from Bullet cluster. $y_{\phi}$ has been fixed from observed relic abundance.

\begin{tabular}{ccc}
\hline \hline $\begin{array}{c}m_{\chi} \\
(\mathrm{GeV})\end{array}$ & $\begin{array}{c}m_{\phi} \\
(\mathrm{MeV})\end{array}$ & $y_{\phi}$ \\
\hline \hline 1 & 4 & 0.06 \\
5 & 9 & 0.11 \\
10 & 15 & 0.15 \\
15 & 20 & 0.18 \\
20 & 30 & 0.2 \\
50 & 60 & 0.32 \\
\hline \hline
\end{tabular}

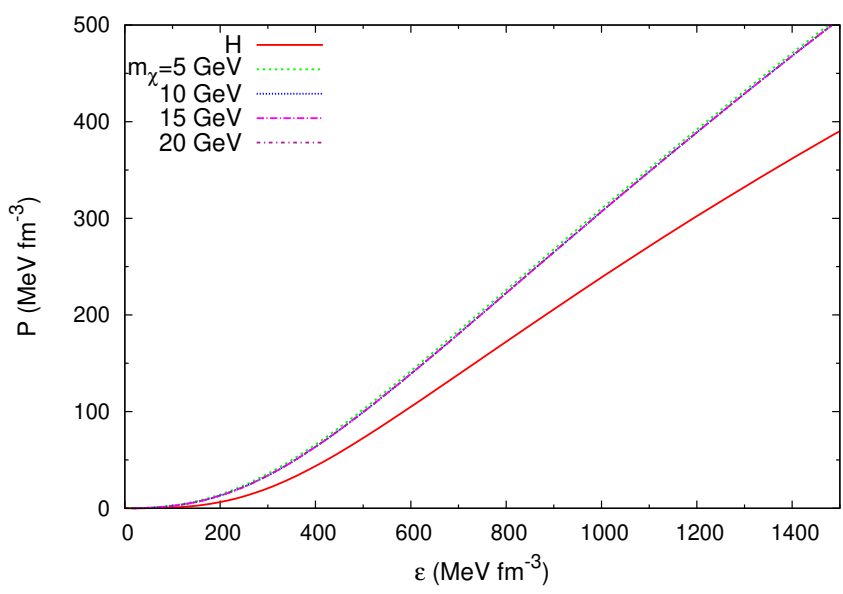

Figure 2. Equation of state of neutron star with $\beta$ stable matter without dark matter $(\mathrm{H})$ and dark matter admixed neutron star matter for different values of $m_{\chi}$.

case where massive $(200 \mathrm{GeV}) \mathrm{DM}$ is chosen as a possible constituent of NS and consequently the EoS softens compared to the no-DM case Panotopoulos \& Lopes (2017), we find that comparatively lighter and feeble interaction of DM with the nucleons stiffens the EoS considerably. This stiffening of EoS in presence of DM can be attributed to the fact that light and the feeble interaction between the DM particles with the nucleons do not affect the nucleon momenta and particle population much and therefore the EoS for the hadronic sector is very less affected while the total energy density and pressure increase due to DM contribution to them as seen in eqs. 15 and 16. To understand this fact better we show in figure 3 the relative abundance of different particles in the NSM including DM.

The neutron fraction reduces as protons, electrons and muon concentration increase, guided by the chemical equilibrium and charge neutrality conditions for $\beta$ equilibrium. The DM fraction reduces gradually towards the core and the hadronic matter population remains almost unaffected by the presence of DM swallowed during the formation of NS. This

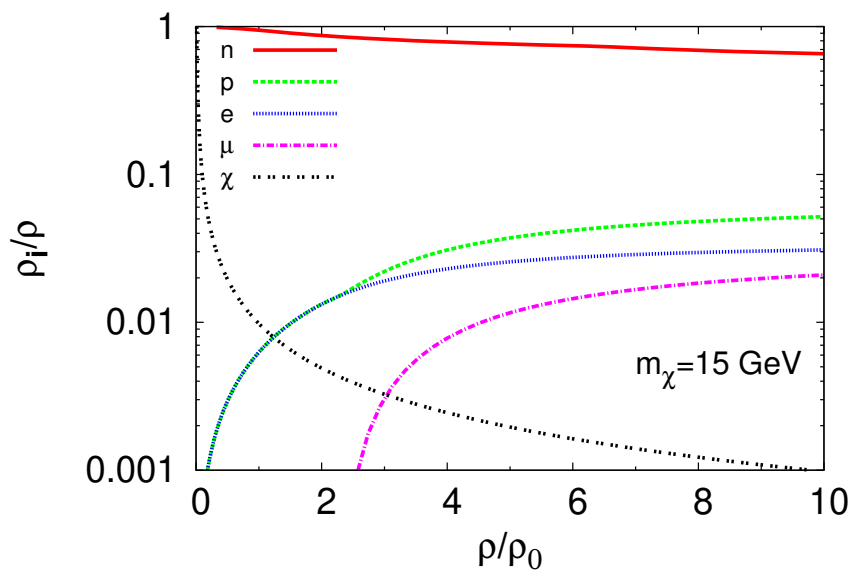

Figure 3. Relative particle population of dark matter admixed neutron star matter for $m_{\chi}=15 \mathrm{GeV}$.

happens due to the consideration that DM has a constant number density throughout the NS. The correspondence is justified by the presence of the dense DM halo as one move outwards NS Li et al. (2012b). Inside the core, at a particular density, moderate fraction of DM adds to the net pressure without affecting the contribution from the hadronic sector and therefore we observe stiffening of the EoS in presence of DM. The density of the DM halo is dependent on the environment at which the NS is formed and is predicted by N-body simulations via NFW profile of various galaxies and clusters Navarro et al. (1996).

The structural properties like gravitational mass, radius and surface redshift of DM admixed NS are computed next for the obtained EoS for different values of $m_{\chi}$. In figure 4 we present the variation of gravitational mass $M$ with respect to radius $R$. We have also compared the case when pure hadronic matter is considered without DM (' $H$ ').

As the EoS stiffens with the inclusion of DM, the effect is also evident from the mass-radius relationship. The maximum gravitational mass has increased considerably when the contribution of DM is considered for any given value of $m_{\chi}$ compared to the pure hadronic case (' $\mathrm{H}$ '). It is also seen that lighter DM fermions contribute more to both mass and radius of NS. For every value of $m_{\chi}$ considered, the maximum mass constraints from the massive pulsars like PSR J0348+0432 Antoniadis et al. (2013) and PSR J0740+6620 Cromartie et al. (2019) are satisfied with the DM admixed NS EoS. It is noteworthy that the pure hadronic EoS alone in absence of DM could not satisfy the maximum mass constraints from these two massive pulsars. Thus the consideration of DM contribution in NS could successfully yield NS configurations compatible with massive pulsar observations. This implies that the model parameter set (shown in table 3 ), that was ruled out (despite satisfying the SNM properties reasonably well) because it could not yield high mass NS 


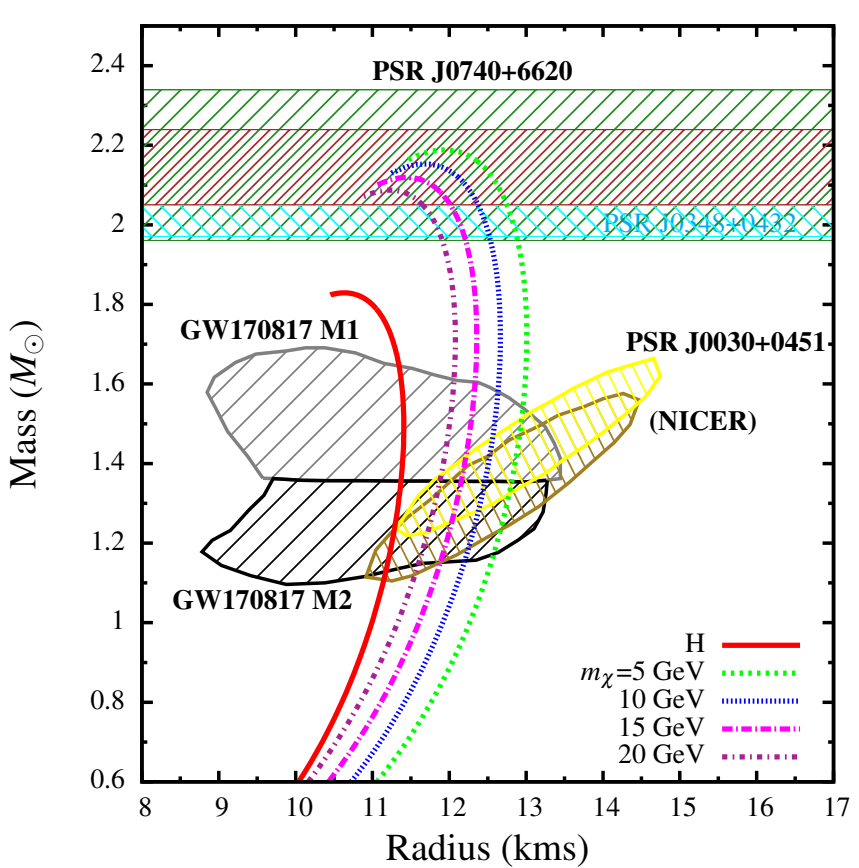

Figure 4. Mass-radius relationship of static neutron stars with $\beta$ stable matter without dark matter $(\mathrm{H})$ and dark matter admixed neutron star matter for different values of $m_{\chi}$. Observational limits imposed from high mass pulsars like PSR J0348+0432 $\left(M=2.01 \pm 0.04 M_{\odot}\right)$ Antoniadis et al. (2013) (cyan shaded region) and PSR J0740+6620 $\left(2.14_{-0.09}^{+0.10} M_{\odot}(68.3 \%\right.$ - brown shaded region) and $2.14_{-0.18}^{+0.20} \quad M_{\odot}(95.4 \%$ - dark green shaded region) $)$ Cromartie et al. (2019) are also indicated. The limit on $R_{1.4}$ Abbott et al. (2017, 2018); Fattoyev et al. (2018) prescribed from GW170817 are indicated by the black horizontal line with arrows. The constraints on $M-R$ plane from NICER experiment for PSR J0030+0451 are also compared (golden shaded region Riley et al. (2019) and yellow shaded region Miller et al. (2019).

configurations, can be revived by considering the presence of light and feebly interacting DM as a possible constituent of NS. Moreover, we also found that the NICER data for mass and radius of PSR J0030+0451 Riley et al. (2019); Miller et al. (2019) are better satisfied with all the values of $m_{\chi}$ considered in the present work compared to the pure hadronic matter case (figure 4 ). The bound on $R_{1.4}$ from GW170817 Abbott et al. (2017, 2018); Fattoyev et al. (2018) is well satisfied for all the cases (with and without DM). In this work we are interested to show that appropriately small values of fermionic DM mass can help to obtain reasonable NS configurations. However, we have also shown that the higher values of $m_{\chi}$ will led to gradual softening of EoS and consequently reduction in maximum mass, radius and tidal deformability of NSs (table 3). Therefore considering $m_{\chi}$ as high as $200 \mathrm{GeV}$ will inevitably soften the EoS and low mass NS compared to the no-DM case as shown in Panotopoulos \& Lopes (2017) and other works.

The variation of surface redshift $Z_{s}$ with respect to gravitational mass for DM admixed NS is depicted in figure 5 for the three values of $m_{\chi}$.

There is considerable increase in the value of maximum surface redshift when DM is considered compared to the pure hadronic case ('H'). There is feeble increase in the value of

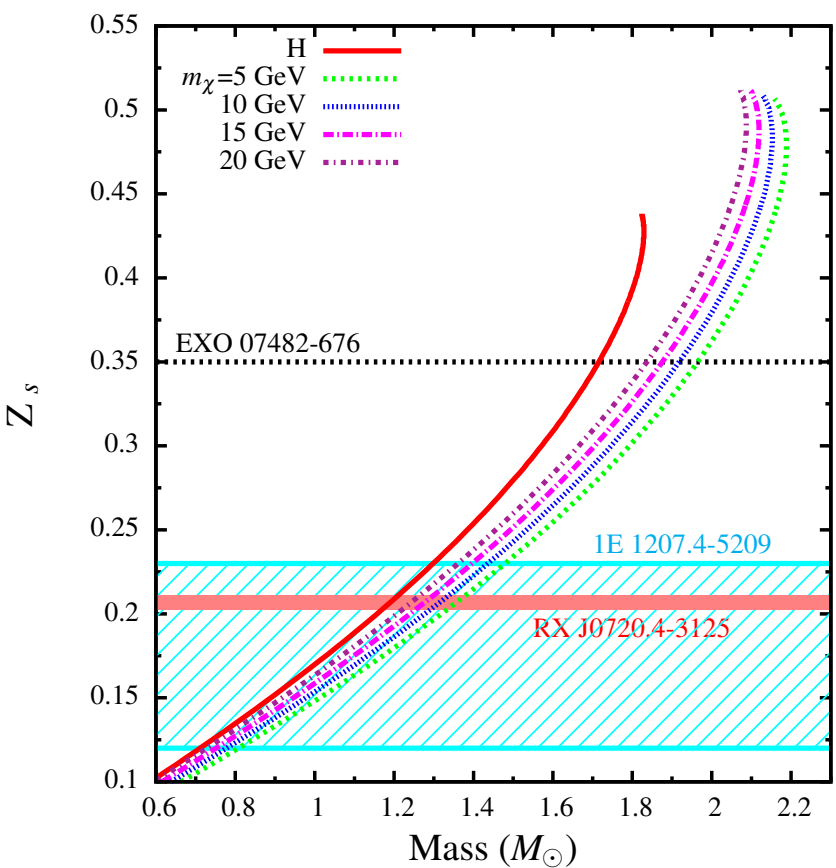

Figure 5. Surface gravitational redshift $Z_{s}$ vs gravitational mass $M$ of dark matter admixed neutron star for different values of $m_{\chi}$. Observational limits imposed from EXO 07482-676 $\left(Z_{S}=0.35\right)$ Cottam et al. (2002), $1 \mathrm{E} 1207.4-5209\left(Z_{S}=(0.12-0.23)\right)$ Sanwal et al. $(2002)$ and RX J0720.4-3125 $\left(Z_{S}=0.205_{-0.003}^{+0.006}\right.$ Hambaryan et al. (2017) are also indicated.

maximum $Z_{s}$ with the variation of $m_{\chi}$. Although $m_{\chi}=100 \mathrm{MeV}$ and $1 \mathrm{GeV}$ yield the same value of maximum mass but the maximum redshift for the latter is slightly greater than that of the former. This is because along with the mass of NS, the surface redshift is also dependent on the value of radius which is slightly less in the latter case i.e., $m_{\chi}=1 \mathrm{GeV}$. The maximum surface redshift constraints from EXO 07482-676 Cottam et al. (2002), 1E 1207.4-5209 Sanwal et al. (2002) and RX J0720.4-3125 Hambaryan et al. (2017) are well satisfied with both DM admixed and pure hadronic NS EoS.

We next calculate the dimensionless tidal deformability $\Lambda=\frac{2}{3} k_{2}\left(\frac{M}{R}\right)^{-5}$ and present our results in figure 6 . For a spherically symmetric star, physical significance of the tidal deformability lies in the modification of the spacetime metric by a linear $l=2$ perturbation.

As expected, the tidal deformability decreases with increasing mass, indicating that massive stars are less deformed. There are decrements in the values of $\Lambda$ for increasing values of $m_{\chi}$. This is because the compactness $(C \equiv M / R)$, that increases with $m_{\chi}$, affects $\Lambda$ directly. Our estimates of $\Lambda_{1.4}$ are in excellent agreement with the constraint on the same obtained from GW170817 data analysis Abbott et al. (2017, 2018).

In figure 7 we show the variation of tidal deformability parameters $\Lambda_{1}$ and $\Lambda_{2}$ linked to the BNS companion with a high mass $M_{1}$ and a low mass $M_{2}$ associated with GW170817 observation. This result is obtained considering NS both with and without DM. The observed mass range for the BNS companion is $1.1 M_{\odot} \leqslant M_{N S} \leqslant 1.6 M_{\odot}$. To ensure the fact $M_{2}<M_{1}$ for the figure 7 , we used $1.365 M_{\odot} \leqslant M_{1} \leqslant 1.6 M_{\odot}$ and fixed 


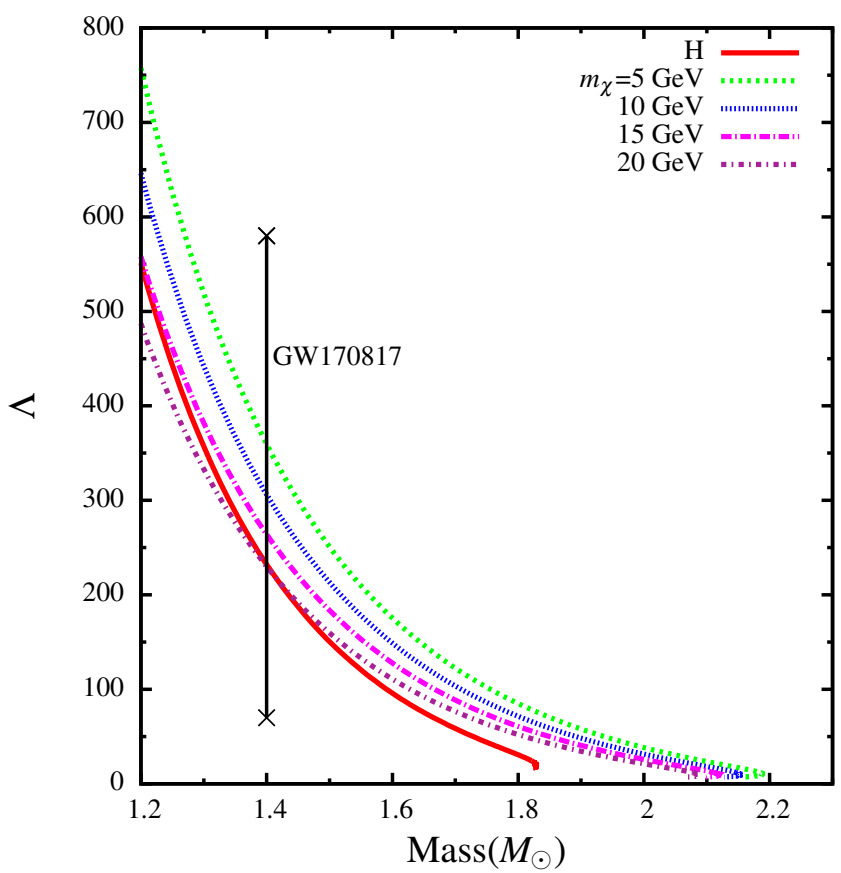

Figure 6. Variation of tidal deformability with respect to gravitational mass of neutron stars with $\beta$ stable matter without dark matter $(\mathrm{H})$ and dark matter admixed neutron star matter for different values of $m_{\chi}$. Constraint on $\Lambda_{1.4}$ from GW170817 observations is also indicated following Abbott et al. (2017, 2018).

$M_{2}$ from the observed chirp mass $M_{\text {chirp }}=1.188 M_{\odot}$ from GW170817 data.

Our estimates of $\Lambda_{1}$ and $\Lambda_{2}$ both in presence and absence of DM are within the bounds specified from GW170817 data analysis Abbott et al. (2017, 2018).

The various structural properties of NS obtained with and without considering DM are tabulated below in table 3 .

The mass of fermionic DM $m_{\chi}$ therefore plays a very significant role in determining the EoS and structural properties of DM admixed NS.

\subsection{Dark matter admixed neutron star for different values of $k_{F}^{X}$}

In order to study the explicit dependence of the EoS and structural properties of NS on the momentum of fermionic DM, we now vary $k_{F}^{\chi}$ by fixing $m_{\chi}=10 \mathrm{GeV}$. The EoS for DM admixed NSM for different values of $k_{F}^{\chi}$ is shown in figure 8 .

Consistent with Panotopoulos \& Lopes (2017) we find that the EoS stiffens with decreasing values of $k_{F}^{\chi}$ which directly affects the EoS as seen from eqs. 15 and 16. The decrease is, however, less in the present work as we have chosen the value of $m_{\chi}$ by satisfying the self-interaction constraint from bullet cluster Randall et al. (2008); Tulin et al. (2013) (as shown in figure 1) and to be quite small compared to that chosen in Panotopoulos \& Lopes (2017). With the obtained EoS, we also calculated the structural properties of DM admixed NS for varying $k_{F}^{\chi}$. Figure 9 shows the variation of gravitational mass with radius for different values of $k_{F}^{X}$.

Both radius and mass increase with decreasing value of $k_{F}^{\chi}$. The maximum gravitational mass constraints from both PSR

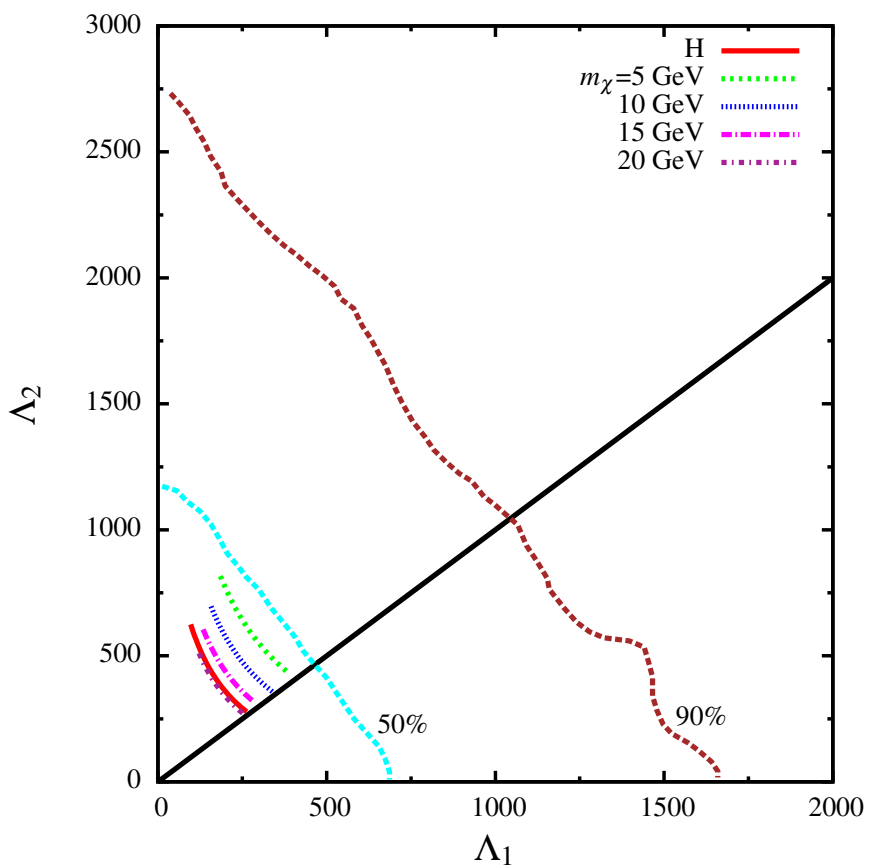

Figure 7. Tidal deformabilities of the individual components of the binary neutron stars associated with GW170817 with $\beta$ stable matter without dark matter $(\mathrm{H})$ and dark matter admixed neutron star matter for different values of $m_{\chi}$. The $50 \%$ and $90 \%$ confidence limits for this event are also indicated following Abbott et al. (2017, 2018).

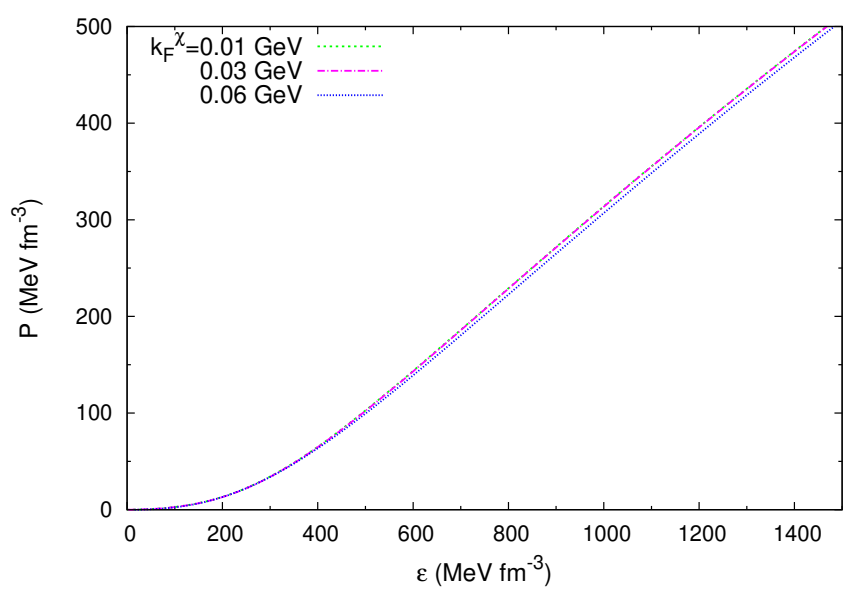

Figure 8. Equation of state of dark matter admixed neutron star matter for different values of $k_{F}^{\chi}$.

J0348+0432 Antoniadis et al. (2013) and PSR J0740+6620 Cromartie et al. (2019) are satisfied with all the chosen values of $k_{F}^{\chi}$ while the $M-R$ results for all the values of $k_{F}^{\chi}$ are in excellent agreement with the NICER data for PSR J0030+0451 Riley et al. (2019); Miller et al. (2019). Also the estimates of $R_{1.4}$ are in agreement with the GW170817 data for BNSM Abbott et al. (2017, 2018); Fattoyev et al. (2018).

In figure 10 we show the variation of $\Lambda$ with respect to mass for different values of $k_{F}^{\chi}$.

We found that as the EoS stiffens with decreasing values 
Table 3. Structural properties of static neutron stars with $\beta$ stable matter without dark matter $(\mathrm{H})$ and dark matter admixed neutron star matter $(\mathrm{H}+\mathrm{DM})$ for different values of $m_{\chi}$.

\begin{tabular}{ccccccc}
\hline \hline & $\begin{array}{c}M_{\max } \\
\left(M_{\odot}\right)\end{array}$ & $\begin{array}{c}R \\
(\mathrm{~km})\end{array}$ & $\begin{array}{c}R_{1.4} \\
(\mathrm{~km})\end{array}$ & $\Lambda_{1.4}$ & $Z_{s}$ & \\
\hline $\mathrm{H}$ & 1.83 & 10.62 & 11.39 & 233.50 & 0.438 & \\
\hline \hline $\mathrm{H}+\mathrm{DM}$ & $m_{\chi}$ & $M_{\max }$ & $\begin{array}{c}R \\
\left(M_{\odot}\right)\end{array}$ & $\begin{array}{c}R_{1.4} \\
(\mathrm{~km})\end{array}$ & $\Lambda_{1.4}$ & $Z_{s}$ \\
& 0.1 & 2.22 & 12.20 & 13.24 & 428.05 & 0.503 \\
& 1 & 2.22 & 12.18 & 13.18 & 394.33 & 0.504 \\
& 5 & 2.19 & 11.93 & 12.86 & 346.99 & 0.507 \\
& 10 & 2.15 & 11.67 & 12.50 & 310.45 & 0.509 \\
& 15 & 2.12 & 11.45 & 12.21 & 256.92 & 0.511 \\
& 20 & 2.09 & 11.23 & 11.92 & 231.96 & 0.513 \\
& 50 & 1.92 & 10.18 & 10.69 & 112.59 & 0.518 \\
\hline \hline
\end{tabular}

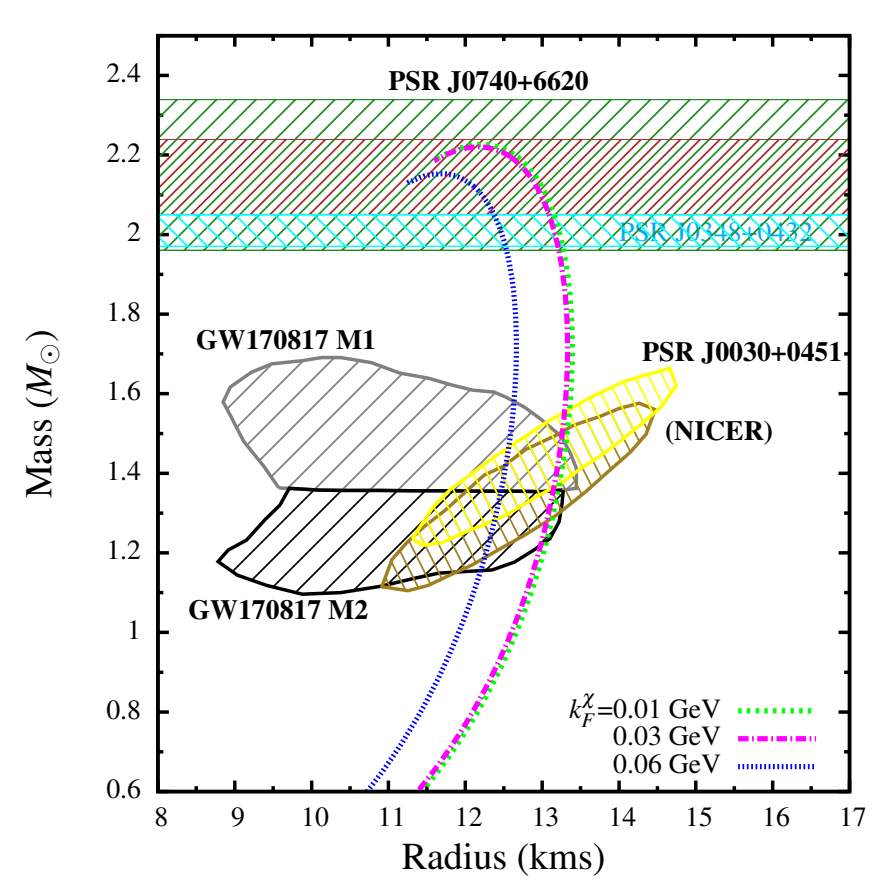

Figure 9. Mass-radius relationship of static neutron stars with dark matter admixed neutron star matter for different values of $k_{F}^{\chi}$. The various constraints shown are same as figure 4 .

Table 4. Structural properties of static neutron stars with dark matter admixed neutron star matter for different values of $k_{F}^{\chi}$.

\begin{tabular}{ccccc}
\hline \hline$k_{F}^{X}$ & $M_{\max }$ & $R$ & $R_{1.4}$ & $\Lambda_{1.4}$ \\
$(\mathrm{GeV})$ & $\left(M_{\odot}\right)$ & $(\mathrm{km})$ & $(\mathrm{km})$ & \\
\hline 0.01 & 2.23 & 12.23 & 13.25 & 427.34 \\
0.03 & 2.22 & 12.17 & 13.18 & 393.12 \\
0.06 & 2.15 & 11.67 & 12.50 & 310.45 \\
\hline \hline
\end{tabular}

of $k_{F}^{\chi}, \Lambda$ increases and the value of $\Lambda_{1.4}$ for all the considered values of $k_{F}^{\chi}$ are consistent with the bound obtained from GW170817 data analysis Abbott et al. (2017, 2018).

Below we tabulate in table 4 the estimates of various structural properties for different values of $k_{F}^{\chi}$.

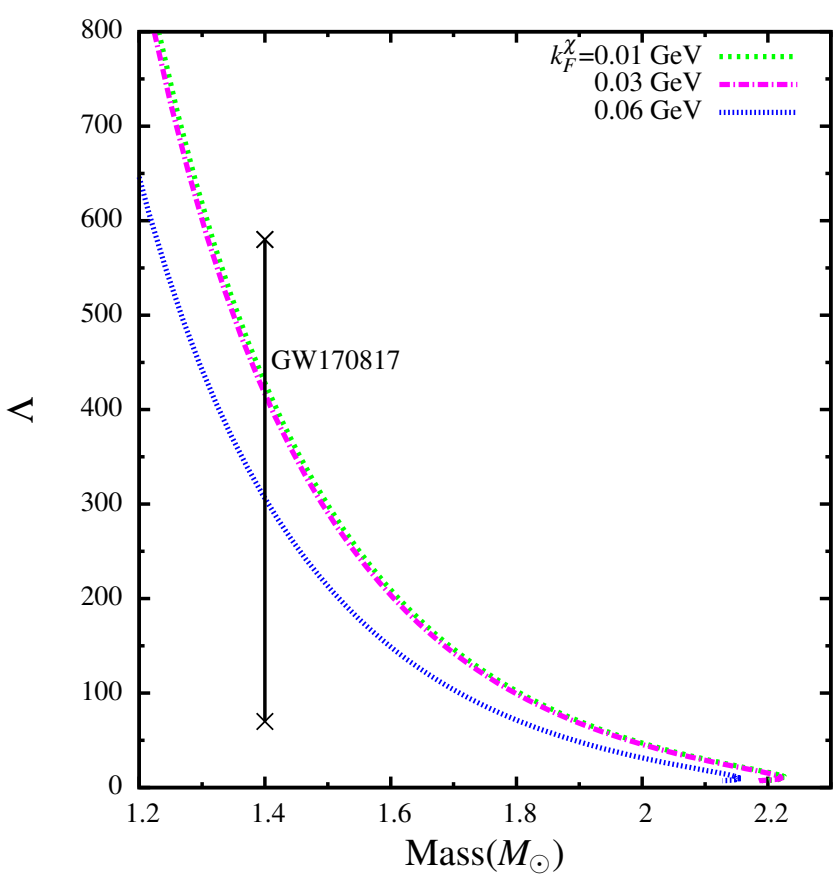

Figure 10. Variation of tidal deformability with respect to gravitational mass of neutron stars with dark matter admixed neutron star matter for different values of $k_{F}^{\chi}$. Constraint on $\Lambda_{1.4}$ from GW170817 observations is also indicated following Abbott et al. (2017, 2018).

\subsection{Enclosed Mass vs Radius Profiles for different values of $k_{F}^{\chi}$ and $m_{\chi}$}

The enclosed mass-radius profile for a chosen value of total NS mass Molla et al. (2020); Del Popolo et al. (2020); Bhat \& Paul (2020) can be obtained by solving the TOV equations Tolman (1939); Oppenheimer \& Volkoff (1939) following Molla et al. (2020). In the figure 11, we show the enclosed mass profiles as a function of radius for NS mass $M=1.0 M_{\odot}, 1.4 M_{\odot}, 2.0 M_{\odot}$ for different values of $k_{F}^{\chi}$ and $m_{\chi}$.

\section{SUMMARY AND CONCLUSIONS}

We consider feebly interacting, thermal DM as a possible constituent of $\beta$ equilibrated NSM. The DM-SM interaction is 

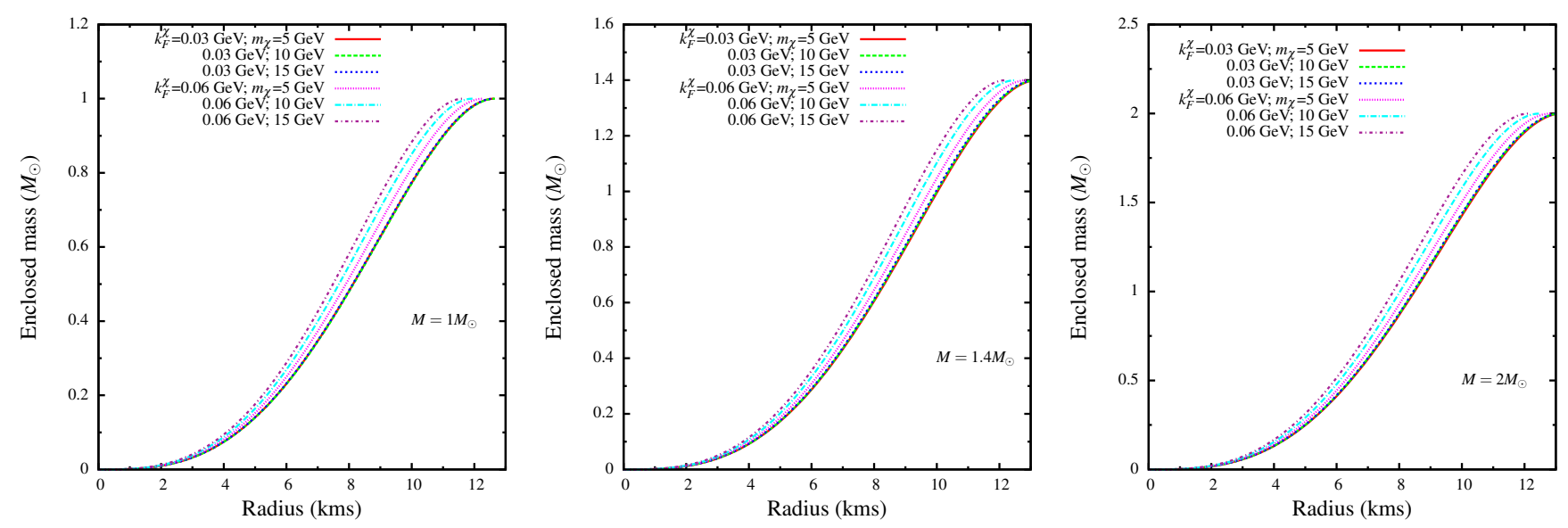

Figure 11. Enclosed Mass-radius profile of static neutron stars with dark matter admixed neutron star matter for different values of $k_{F}^{\chi}$ and $m_{\chi}$. Mass of the NS has been fixed to $M=1.0 M_{\odot}, 1.4 M_{\odot}, 2.0 M_{\odot}$, respectively.

invoked though light new physics mediator whose mass $m_{\phi}$ and coupling $y_{\phi}$ are relate to the mass of fermionic DM $m_{\chi}$ via the self-interaction constraint from the Bullet cluster and from the present day relic abundance. We show that a suitable choice of low $\mathrm{GeV} m_{\chi}$, consistent with the constraints from Bullet cluster, can stiffen the overall EoS. With such consideration, even a soft and ruled out pure hadronic EoS could be successfully revived to obtain static NS structural properties that satisfy the various constraints on NS properties. For all the considered values of $m_{\chi}$, the maximum mass obtained is consistent with the limits imposed from massive pulsars like PSR J0348+0432 and PSR J0740+6620. Our results of $R_{1.4}$ and $\Lambda_{1.4}, \Lambda_{1}$ and $\Lambda_{2}$ fall within the individual range prescribed by data analysis of GW170817. Moreover, the consideration of DM also helped to satisfy the massradius constraints from NICER experiment. Also our estimates of maximum surface redshift are in accordance with the bounds obtained from EXO 07482-676, 1E 1207.4-5209 and RX J0720.4-3125. It is seen that the presence of massive DM fermion reduces the maximum mass, radius and tidal deformability of the NS.

Since we considered constant density distribution of DM, we also varied the DM Fermi momentum to ensure that for a given low $\mathrm{GeV}$ mass value of DM fermion, we obtain stiffer EoS with different values of $k_{F}^{\chi}$ compared to the no-DM case and consequently reasonable values the maximum mass, radius and tidal deformability compatible with bounds obtained on them from the massive pulsars and GW170817 data.

\section{APPENDIX A: ESTIMATION OF $g_{\phi N N}$}

For the new scalar mediator $\phi$-quark interaction

$$
\mathcal{L}_{\phi, q}=\sum_{q} g_{q} \bar{q} q \phi=\left(\sqrt{2} G_{F}\right)^{1 / 2} \sum_{q} \epsilon_{q} m_{q} \bar{q} q \phi
$$

Here, $G_{F}$ is the Fermi constant and the parameter $\epsilon_{q}=1$ for all quarks in SM but we can vary $\epsilon_{q}$ for BSM physics Cheng \& Chiang (2012).

For nucleons the effective interaction $g_{\phi N N} \bar{N} N \phi$ with

$$
g_{\phi N N}=\left(\sqrt{2} G_{F}\right)^{1 / 2} \sum_{q}<N\left|\epsilon_{q} m_{q} \bar{q} q\right| N>
$$

Now

$$
\begin{array}{r}
<N|\bar{q} q| N>=\frac{m_{N}}{m_{q}} f_{N}^{S q} \quad \text { for } \mathrm{u}, \mathrm{d}, \mathrm{s} \\
<N|\bar{q} q| N>=\frac{2}{27} \frac{m_{N}}{m_{q}} f_{N}^{S G} \quad \text { for c, b, t }
\end{array}
$$

We can estimate $g_{\phi N N}$ with one free parameter $\epsilon_{q}$, whereas, we have the following standard values from lattice QCD simulation data and chiral perturbation theory Backović et al. (2015)

$$
\begin{aligned}
& f_{p}^{S u}=0.0153 ; f_{p}^{S d}=0.0191 ; f_{p}^{S s}=0.0447 \\
& f_{n}^{S u}=0.0110 ; f_{n}^{S d}=0.0273 ; f_{n}^{S s}=0.0447 \\
& f_{N}^{S G}=1-\sum_{q} f_{N}^{S q}
\end{aligned}
$$

Estimated value of $g_{\phi N N}$ to be

$$
g_{\phi N N} \leqslant 1.1 \times 10^{-4}
$$

\section{DATA AVAILABILITY}

The data underlying this article are available within the article.

\section{REFERENCES}

Aaboud M., et al., 2018a, JHEP, 10, 031

Aaboud M., et al., 2018b, Phys. Rev. D, 97, 052012

Aaboud M., et al., 2019a, Eur. Phys. J. C, 79, 481

Aaboud M., et al., 2019b, Phys. Rev. D, 99, 012001

Aaboud M., et al., 2019c, Phys. Rev. D, 99, 052005

Aaboud M., et al., 2019d, Phys. Rev. Lett., 122, 151801

Aaboud M., et al., 2019e, Phys. Lett. B, 788, 96

Aad G., et al., 2015, Phys. Rev. D, 92, 072004

Aad G., et al., 2020a, Eur. Phys. J. C, 80, 450

Aad G., et al., 2020b, Phys. Rev. D, 101, 052013

Aad G., et al., 2020c, Phys. Rev. D, 102, 032006

Aad G., et al., 2020d, Phys. Lett. B, 801, 135114

Aaij R., et al., 2021, Eur. Phys. J. C, 81, 261

Aalseth C. E., et al., 2008, Phys. Rev. Lett., 101, 251301

Aalseth C. E., et al., 2009, Phys. Rev. Lett., 102, 109903

Aartsen M. G., et al., 2014, Phys. Rev. Lett., 113, 101101 
Abbott B. P., et al., 2017, Phys. Rev. Lett., 119, 161101 Abbott B. P., et al., 2018, Phys. Rev. Lett., 121, 161101 Abdallah H., et al., 2016, Phys. Rev. Lett., 117, 111301 Accardo L., et al., 2014, Phys. Rev. Lett., 113, 121101 Ade P. A. R., et al., 2014, Astron. Astrophys., 571, A16 Adriani O., et al., 2009, Nature, 458, 607

Adriani O., et al., 2013, Phys. Rev. Lett., 111, 081102

Adriani O., et al., 2017, Phys. Rev. Lett., 119, 181101

Adriani O., et al., 2018, Phys. Rev. Lett., 120, 261102

Aghanim N., et al., 2020, Astron. Astrophys., 641, A6

Agnes P., et al., 2018, Phys. Rev. Lett., 121, 111303

Agnese R., et al., 2018, Phys. Rev. Lett., 121, 051301

Aguilar M., et al., 2014, Phys. Rev. Lett., 113, 121102

Akerib D. S., et al., 2013, Astropart. Phys., 45, 34

Alcantara E., Anchordoqui L. A., Soriano J. F., 2019, Phys. Rev. D, 99, 103016

Ali-Haïmoud Y., Chluba J., Kamionkowski M., 2015, Phys. Rev. Lett., 115, 071304

Alimena J., et al., 2020, J. Phys. G, 47, 090501

Alvarez-Castillo D. E., Blaschke D. B., Grunfeld A. G., Pagura V. P., 2019, Phys. Rev. D, 99, 063010

Ambrosi G., et al., 2017, Nature, 552, 63

Andreas S., Hambye T., Tytgat M. H. G., 2008, JCAP, 10, 034

Angloher G., et al., 2016, Eur. Phys. J. C, 76, 25

Antoniadis J., et al., 2013, Science, 340, 6131

Aprile E., et al., 2012, Phys. Rev. Lett., 109, 181301

Aprile E., et al., 2018, Phys. Rev. Lett., 121, 111302

Aprile E., et al., 2020, Phys. Rev. D, 102, 072004

Atwood W. B., et al., 2009, Astrophys. J., 697, 1071

Backović M., Martini A., Mattelaer O., Kong K., Mohlabeng G., 2015, Phys. Dark Univ., 9-10, 37

Baltz E. A., 2004, eConf, C040802, L002

Barbieri R., Curci G., 1989, Phys. Lett. B, 219, 503

Bauer M., Plehn T., 2019, Yet Another Introduction to Dark Matter: The Particle Physics Approach. Lecture Notes in Physics Vol. 959, Springer (arXiv: 1705. 01987), doi:10.1007/978-3-03016234-4

Bauer J. B., Marsh D. J. E., Hložek R., Padmanabhan H., Laguë A., 2020, Mon. Not. Roy. Astron. Soc., 500, 3162

Belanger G., Boudjema F., Pukhov A., Semenov A., 2014, Comput. Phys. Commun., 185, 960

Bennett C. L., et al., 2013, Astrophys. J. Suppl., 208, 20

Bernabei R., et al., 2008, Eur. Phys. J. C, 56, 333

Bernal T., Fernández-Hernández L. M., Matos T., Rodríguez-Meza M. A., 2018, Mon. Not. Roy. Astron. Soc., 475, 1447

Bertone G., Hooper D., Silk J., 2005, Phys. Rept., 405, 279

Bertoni B., Nelson A. E., Reddy S., 2013, Phys. Rev. D, 88, 123505

Bhat S. A., Paul A., 2020, Eur. Phys. J. C, 80, 544

Bhattacherjee B., Mukherjee S., Sengupta R., Solanki P., 2020, JHEP, 08, 141

Bionta R. M., et al., 1987, Phys. Rev. Lett., 58, 1494

Blekman F., Desai N., Filimonova A., Sahasransu A. R., Westhoff S., 2020, JHEP, 11, 112

Boudaud M., Lavalle J., Salati P., 2017, Phys. Rev. Lett., 119, 021103

Bradac M., et al., 2006, Astrophys. J., 652, 937

Bravin M., et al., 1999, Astropart. Phys., 12, 107

Cannoni M., 2016, Eur. Phys. J. C, 76, 137

Carr J., et al., 2016, PoS, ICRC2015, 1203

Chang J., et al., 2008, Nature, 456, 362

Cheng H.-Y., Chiang C.-W., 2012, JHEP, 07, 009

Ciarcelluti P., Sandin F., 2011, Phys. Lett. B, 695, 19

Cottam J., Paerels F., Mendez M., 2002, Nature, 420, 51

Crisler M., Essig R., Estrada J., Fernandez G., Tiffenberg J., Sofo haro M., Volansky T., Yu T.-T., 2018, Phys. Rev. Lett., 121,061803

Cromartie H. T., et al., 2019, Nature Astron., 4, 72

Danielewicz P., Lacey R., Lynch W. G., 2002, Science, 298, 1592
Dave R., Spergel D. N., Steinhardt P. J., Wandelt B. D., 2001, Astrophys. J., 547, 574

Davoudiasl H., Murphy C. W., 2017, Phys. Rev. Lett., 118, 141801

Dawson W. A., et al., 2012, Astrophys. J. Lett., 747, L42

Del Popolo A., Le Delliou M., Deliyergiyev M., 2020, Universe, 6, 222

Deliyergiyev M., Del Popolo A., Tolos L., Le Delliou M., Lee X., Burgio F., 2019, Phys. Rev. D, 99, 063015

Dreiner H. K., Fortin J.-F., Hanhart C., Ubaldi L., 2014, Phys. Rev. D, 89, 105015

Du N., et al., 2018, Phys. Rev. Lett., 120, 151301

Dutra M., et al., 2014, Phys. Rev. C, 90, 055203

Ellis J., Hütsi G., Kannike K., Marzola L., Raidal M., Vaskonen V., 2018, Phys. Rev. D, 97, 123007

Elsaesser D., Mannheim K., 2005, New Astron. Rev., 49, 297

Essig R., Manalaysay A., Mardon J., Sorensen P., Volansky T., 2012, Phys. Rev. Lett., 109, 021301

Fattoyev F. J., Piekarewicz J., Horowitz C. J., 2018, Phys. Rev. Lett., 120, 172702

Garg U., Colò G., 2018, Prog. Part. Nucl. Phys., 101, 55

Gondolo P., Gelmini G., 1991, Nucl. Phys. B, 360, 145

Guha A., J S., Das P. K., 2017, Phys. Rev. D, 95, 015001

Guha A., Dev P. S. B., Das P. K., 2019, JCAP, 02, 032

Hambaryan V., Suleimanov V., Haberl F., Schwope A. D., Neuhäuser R., Hohle M., Werner K., 2017, Astron. Astrophys., 601, A108

Hambye T., Vanderheyden L., 2020, JCAP, 05, 001

Harding J. P., Dingus B., 2016, PoS, ICRC2015, 1227

Hinderer T., 2008, Astrophys. J., 677, 1216

Hinderer T., Lackey B. D., Lang R. N., Read J. S., 2010, Phys. Rev. D, 81, 123016

Hirata K., et al., 1987, Phys. Rev. Lett., 58, 1490

Horowitz C. J., Serot B. D., 1981, Nucl. Phys. A, 368, 503

Janka H. T., 2017, Alsabti A., Murdin P. (eds) Handbook of Supernovae. Springer, Cham

Janka H.-T., Langanke K., Marek A., Martinez-Pinedo G., Mueller B., 2007, Phys. Rept., 442, 38

Jha T. K., Mishra H., 2008, Phys. Rev. C, 78, 065802

Jha T. K., Raina P. K., Panda P. K., Patra S. K., 2006, Phys. Rev. C, 74, 055803

Jha T. K., Mishra H., Sreekanth V., 2008, Phys. Rev. C, 77, 045801

Kadota K., Silk J., 2014, Phys. Rev. D, 89, 103528

Kahlhoefer F., Schmidt-Hoberg K., Kummer J., Sarkar S., 2015, Mon. Not. Roy. Astron. Soc., 452, L54

Khachatryan V., et al., 2016, Phys. Rev. D, 94, 112004

Khan E., Margueron J., 2012, Phys. Rev. Lett., 109, 092501

Khan E., Margueron J., 2013, Phys. Rev. C, 88, 034319

Kolb E. W., Long A. J., 2017, Phys. Rev. D, 96, 103540

Kolb E. W., Starobinsky A. A., Tkachev I. I., 2007, JCAP, 07, 005

Krnjaic G., 2016, Phys. Rev. D, 94, 073009

Kurkela A., Fraga E. S., Schaffner-Bielich J., Vuorinen A., 2014, Astrophys. J., 789, 127

Lee H. S., et al., 2014, Phys. Rev. D, 90, 052006

Li X., Wang F., Cheng K. S., 2012a, JCAP, 10, 031

Li A., Huang F., Xu R.-X., 2012b, Astropart. Phys., 37, 70

Miller M. C., et al., 2019, Astrophys. J. Lett., 887, L24

Molla S., Ghosh B., Kalam M., 2020, Eur. Phys. J. Plus, 135, 819

Mukhopadhyay S., Atta D., Imam K., Basu D. N., Samanta C., 2017, Eur. Phys. J. C, 77, 440

Navarro J. F., Frenk C. S., White S. D. M., 1996, Astrophys. J., 462,563

Nelson A., Reddy S., Zhou D., 2019, JCAP, 07, 012

Ni Y., Wang M.-Y., Feng Y., Di Matteo T., 2019, Mon. Not. Roy. Astron. Soc., 488, 5551

Oppenheimer J. R., Volkoff G. M., 1939, Phys. Rev., 55, 374

Panotopoulos G., Lopes I., 2017, Phys. Rev. D, 96, 083004

Perez-Garcia M. A., Silk J., Stone J. R., 2010, Phys. Rev. Lett., 105, 141101 
Raffelt G. G., 1996, Stars as laboratories for fundamental physics: The astrophysics of neutrinos, axions, and other weakly interacting particles. University of Chicago Press

Raithel C., Özel F., Psaltis D., 2018, Astrophys. J. Lett., 857, L23

Randall S. W., Markevitch M., Clowe D., Gonzalez A. H., Bradac M., 2008, Astrophys. J., 679, 1173

Razeira M., Mesquita A., Vasconcellos C. A. Z., Gomes R. O., 2011, Int. J. Mod. Phys. E, 20, 109

Rezaei Z., 2017, Astrophys. J., 835, 33

Riley T. E., et al., 2019, Astrophys. J. Lett., 887, L21

Robertson A., Massey R., Eke V., 2017, Mon. Not. Roy. Astron. Soc., 465, 569

Sahu P. K., Ohnishi A., 2000, Prog. Theor. Phys., 104, 1163

Sanwal D., Pavlov G. G., Zavlin V. E., Teter M. A., 2002, Astrophys. J. Lett., 574, L61

Sen D., 2021, J. Phys. G, 48, 025201

Sen D., Jha T. K., 2019, J. Phys. G, 46, 015202

Shapiro S. L., Teukolsky S. A., 1983, Black holes, white dwarfs, and neutron stars: The physics of compact objects. Wiley-VCH, Berlin

Sirunyan A. M., et al., 2018a, JHEP, 08, 016

Sirunyan A. M., et al., 2018b, Phys. Rev. D, 98, 092011
Sirunyan A. M., et al., 2019a, Phys. Rev. D, 99, 032011 Sirunyan A. M., et al., 2019b, Phys. Rev. D, 99, 032014 Sirunyan A. M., et al., 2019c, Phys. Rev. D, 100, 112003 Sirunyan A. M., et al., 2019d, Phys. Lett. B, 797, 134876 Sirunyan A. M., et al., 2020a, Eur. Phys. J. C, 80, 3 Sirunyan A. M., et al., 2020b, Phys. Lett. B, 806, 135502 Stone J. R., Reinhard P. G., 2007, Prog. Part. Nucl. Phys., 58, 587 Sumner T. J., 2005, New Astron. Rev., 49, 277

Tanabashi M., et al., 2018, Phys. Rev. D, 98, 030001

Tolman R. C., 1939, Phys. Rev., 55, 364

Tolos L., Schaffner-Bielich J., 2015, Phys. Rev. D, 92, 123002

Tremaine S., Gunn J. E., 1979, Phys. Rev. Lett., 42, 407

Tulin S., Yu H.-B., 2018, Phys. Rept., 730, 1

Tulin S., Yu H.-B., Zurek K. M., 2013, Phys. Rev. D, 87, 115007

Vogelsberger M., Zavala J., Loeb A., 2012, Mon. Not. Roy. Astron. Soc., 423,3740

Wang Q., et al., 2020, Chin. Phys. C, 44, 125001

Zhu Z.-Y., Zhou E.-P., Li A., 2018, Astrophys. J., 862, 98

Zitzer B., 2018, PoS, ICRC2017, 904

Zyla P. A., et al., 2020, PTEP, 2020, 083C01

de Lavallaz A., Fairbairn M., 2010, Phys. Rev. D, 81, 123521 